\title{
Some Properties of Third-Order Differential Equations with Mixed Arguments
}

\author{
B. Baculíková and J. Džurina \\ Department of Mathematics, Faculty of Electrical Engineering and Informatics, Technical University of Košice, Letná 9 , \\ 04200 Košice, Slovakia \\ Correspondence should be addressed to B. Baculíková; blanka.baculikova@tuke.sk
}

Received 14 August 2012; Accepted 2 January 2013

Academic Editor: Bruce A. Watson

Copyright (C 2013 B. Baculíková and J. Džurina. This is an open access article distributed under the Creative Commons Attribution License, which permits unrestricted use, distribution, and reproduction in any medium, provided the original work is properly cited.

We offer a new comparison the principle for deducing properties of third-order differential equations with mixed arguments, $\left(r(t)\left[x^{\prime}(t)\right]^{\gamma}\right)^{\prime \prime}+q(t) f(x(\tau(t)))+p(t) h(x(\sigma(t)))=0$, from those of the corresponding differential equations, without deviating arguments. The presented technique permits to extend immediately the results known for an equation without deviating arguments to a more general equation with advanced and delay arguments.

\section{Introduction}

We consider third-order differential equations with mixed arguments

$$
\left(r(t)\left[x^{\prime}(t)\right]^{\gamma}\right)^{\prime \prime}+q(t) f(x(\tau(t)))+p(t) h(x(\sigma(t)))=0
$$

where we assume that $r, q, \tau, p, \sigma \in C\left(\left[t_{0}, \infty\right)\right), f, h \in$ $C((-\infty, \infty))$, and

$\left(H_{1}\right) \gamma$ is the ratio of two positive odd integers,

$\left(H_{2}\right) r(t)>0, q(t)>0$, and $p(t)>0$,

$\left(H_{3}\right) \sigma(t) \geq t, \tau(t) \leq t, \lim _{t \rightarrow \infty} \tau(t)=\infty$, and $\tau^{\prime}(t)>0$,

$\left(H_{4}\right) x f(x)>0, f^{\prime}(x) \geq 0$ for $x \neq 0$, and $-f(-x y) \geq$ $f(x y) \geq f(x) f(y)$ for $x y>0$,

$\left(H_{5}\right) x h(x)>0, h^{\prime}(x) \geq 0$ for $x \neq 0$, and $-h(-x y) \geq$ $h(x y) \geq h(x) h(y)$ for $x y>0$.

In this paper, we study the canonical case of $(E)$, that is,

$$
R(t)=\int_{t_{0}}^{t} r^{-1 / \gamma}(s) \mathrm{d} s \longrightarrow \infty \quad \text { as } t \longrightarrow \infty \text {. }
$$

There is a permanent interest in studying the properties of third-order differential equations. Various techniques have been presented for such equations (see [1-21]). Comparison theorems, especially, are a very strong and effective tool in the oscillation theory. Mahfoud [18] has presented a very useful comparison technique for studying the properties of delay differential equations through those of differential equations without delay. However, the corresponding result for advanced differential equation is still missing. In this paper, we fill up this gap in the oscillation theory; and moreover, we present such a comparison result that works also for the differential equation with mixed arguments.

\section{The Main Results}

The following result is crucial for our intended comparison theorem.

Lemma 1. Assume that $z(t)>0, z^{\prime}(t)>0$, and $\left(r(t)\left[z^{\prime}(t)\right]^{\gamma}\right)^{\prime}>0$, eventually. Then, for arbitrary $k \in(0,1)$,

$$
z[\sigma(t)] \geq k \frac{R(\sigma(t))}{R(t)} z(t)
$$

eventually.

Proof. It follows from the monotonicity of $w(t)=r(t)\left[z^{\prime}(t)\right]^{\gamma}$ that

$$
\begin{aligned}
& z[\sigma(t)]-z(t) \\
& =\int_{t}^{\sigma(t)} z^{\prime}(s) \mathrm{d} s
\end{aligned}
$$




$$
\begin{aligned}
& =\int_{t}^{\sigma(t)} w^{1 / \gamma}(s) r^{-1 / \gamma}(s) \mathrm{d} s \\
& \geq w^{1 / \gamma}(t)(R(\sigma(t))-R(t)),
\end{aligned}
$$

that is,

$$
\frac{z[\sigma(t)]}{z(t)} \geq 1+\frac{w^{1 / \gamma}(t)}{z(t)}[R(\sigma(t))-R(t)] .
$$

On the other hand, since $z(t) \rightarrow \infty$ as $t \rightarrow \infty$, then for any $k \in(0,1)$ there exists a $t_{1}$ large enough, such that

$$
\begin{aligned}
k z(t) & \leq z(t)-z\left(t_{1}\right) \\
& =\int_{t_{1}}^{t} w^{1 / \gamma}(s) r^{-1 / \gamma}(s) \mathrm{d} s \\
& \leq w^{1 / \gamma}(t) \int_{t_{1}}^{t} r^{-1 / \gamma}(s) \mathrm{d} s \\
& =w^{1 / \gamma}(t)\left[R(t)-R\left(t_{1}\right)\right] \\
& \leq w^{1 / \gamma}(t) R(t)
\end{aligned}
$$

or equivalently

$$
\frac{w^{1 / \gamma}(t)}{z(t)} \geq \frac{k}{R(t)}
$$

Using (6) in (4), we obtain that

$$
\frac{z[\sigma(t)]}{z(t)} \geq 1+\frac{k}{R(t)}[R(\sigma(t))-R(t)] \geq k \frac{R(\sigma(t))}{R(t)} .
$$

We introduce the structure of nonoscillatory solutions.

Lemma 2. Every nonoscillatory solution $x(t)$ of $(E)$ satisfies one of the folowing:

$$
\begin{aligned}
& x(t) x^{\prime}(t)<0, \quad x(t)\left[r(t)\left[x^{\prime}(t)\right]^{\gamma}\right]^{\prime}>0, \\
& x(t)\left[r(t)\left[x^{\prime}(t)\right]^{\gamma}\right]^{\prime \prime}<0, \\
& x(t) x^{\prime}(t)>0, \quad x(t)\left[r(t)\left[x^{\prime}(t)\right]^{\gamma}\right]^{\prime}>0, \\
& x(t)\left[r(t)\left[x^{\prime}(t)\right]^{\gamma}\right]^{\prime \prime}<0,
\end{aligned}
$$

eventually.

This structure follows from canonical case of $(E)$, and the proof can be omitted. It is well known that for a particular case of $(E)$, namely, for the equation

$$
x^{\prime \prime \prime}(t)+p(t) x(t)=0,
$$

there always exists a solution satisfying $\left(N_{0}\right)$, and this fact leads to the following definition, which is due to Kiguradze and Chanturia [15].
Definition 3. One can say that $(E)$ enjoys property (A) if all its nonoscillatory solutions $x(t)$ satisfy $\left(N_{0}\right)$.

We are prepared to present the main result.

Theorem 4. Assume that for some $k \in(0,1)$ the differential inequality

$$
\begin{aligned}
& \left\{\left(r(t)\left[z^{\prime}(t)\right]^{\gamma}\right)^{\prime \prime}+\frac{q\left(\tau^{-1}(t)\right)}{\tau^{\prime}\left(\tau^{-1}(t)\right)} f(z(t))\right. \\
& \left.\quad+p(t) h\left(k \frac{R(\sigma(t))}{R(t)}\right) h(z(t))\right\} \operatorname{sgn} z(t) \leq 0
\end{aligned}
$$

enjoys property $(A)$, then so does $(E)$.

Proof. Assume the contrary, that is, we admit that $(E)$ possesses a positive solution $x(t)$ satisfying $\left(N_{2}\right)$. Thus, it follows from Lemma 1 that, for every $k \in(0,1)$,

$$
\begin{aligned}
(r(t) & {\left.\left[x^{\prime}(t)\right]^{\gamma}\right)^{\prime \prime}+q(t) f(x(\tau(t))) } \\
& +p(t) h\left(k \frac{R(\sigma(t))}{R(t)}\right) h(x(t)) \leq 0 .
\end{aligned}
$$

Integrating from $t$ to $\infty$, we obtain that

$$
\begin{aligned}
\left(r(t)\left[x^{\prime}(t)\right]^{\gamma}\right)^{\prime} \\
\geq \int_{t}^{\infty} q(s) f(x(\tau(s))) \mathrm{d} s \\
\quad+\int_{t}^{\infty} p(s) h\left(k \frac{R(\sigma(s))}{R(s)}\right) h(x(s)) \mathrm{d} s \\
=\int_{\tau(t)}^{\infty} \frac{q\left(\tau^{-1}(s)\right)}{\tau^{\prime}\left(\tau^{-1}(s)\right)} f(x(s)) \mathrm{d} s \\
\quad+\int_{t}^{\infty} p(s) h\left(k \frac{R(\sigma(s))}{R(s)}\right) h(x(s)) \mathrm{d} s \\
\geq \int_{t}^{\infty}\left[\frac{q\left(\tau^{-1}(s)\right)}{\tau^{\prime}\left(\tau^{-1}(s)\right)} f(x(s))\right. \\
\left.\quad+p(s) h\left(k \frac{R(\sigma(s))}{R(s)}\right) h(x(s))\right] \mathrm{d} s .
\end{aligned}
$$

Integrating twice from $t_{1}$ to $t$, we are led to

$$
\begin{aligned}
x(t) \geq \int_{t_{1}}^{t} r^{-1 / \gamma}(v) & \\
\times & {\left[\int_{t_{1}}^{v} \int_{u}^{\infty} \frac{q\left(\tau^{-1}(s)\right)}{\tau^{\prime}\left(\tau^{-1}(s)\right)} f(x(s))\right.} \\
& \left.+p(s) h\left(k \frac{R(\sigma(s))}{R(s)}\right) h(x(s)) \mathrm{d} s \mathrm{~d} u\right]^{1 / \gamma} \mathrm{d} v .
\end{aligned}
$$


Let us denote the right-hand side by $z(t)$. Then, $x(t) \geq z(t)$, $z(t)$ satisfies $\left(N_{2}\right)$, and

$$
\begin{aligned}
0= & \left(r(t)\left[z^{\prime}(t)\right]^{\gamma}\right)^{\prime \prime}+\frac{q\left(\tau^{-1}(t)\right)}{\tau^{\prime}\left(\tau^{-1}(t)\right)} f(x(t)) \\
& +p(t) h\left(k \frac{R(\sigma(t))}{R(t)}\right) h(x(t)) \\
\geq & \left(r(t)\left[z^{\prime}(t)\right]^{\gamma}\right)^{\prime \prime}+\frac{q\left(\tau^{-1}(t)\right)}{\tau^{\prime}\left(\tau^{-1}(t)\right)} f(x(t)) \\
& +p(t) h\left(k \frac{R(\sigma(t))}{R(t)}\right) h(z(t)),
\end{aligned}
$$

which contradicts with the assumptions of the theorem, and we conclude that $(E)$ has property $(A)$.

Theorem 4 reduces the examination of properties of differential equations with mixed arguments to that of simpler equations without deviating arguments and permits to extend immediately the criteria known for property (A) of equations without deviating arguments to more general equations with both advanced and delay arguments. We provide some applications of our main result.

For our further references, we set that

$$
\begin{gathered}
Q_{1}(t)=\left[\frac{q\left(\tau^{-1}(s)\right)}{\tau^{\prime}\left(\tau^{-1}(s)\right)}+p(s)\left(k \frac{R(\sigma(s))}{R(t)}\right)^{\gamma}\right], \\
Q(t)=\int_{t}^{\infty} Q_{1}(s) \mathrm{d} s,
\end{gathered}
$$

where $k \in(0,1)$.

Theorem 5. If, for some $k \in(0,1)$,

$$
\liminf _{t \rightarrow \infty} \frac{1}{Q(t)} \int_{t}^{\infty}\left(\frac{s}{r(s)}\right)^{1 / \gamma} Q^{1+1 / \gamma}(s) \mathrm{d} s>\frac{1}{(\gamma+1)^{1+1 / \gamma}}
$$

then

$$
\left(r(t)\left[x^{\prime}(t)\right]^{\gamma}\right)^{\prime \prime}+q(t) x^{\gamma}(\tau(t))+p(t) x^{\gamma}(\sigma(t))=0
$$

enjoys property $(A)$

Proof. By Theorem 4, it is sufficient to show that the differential inequality

$$
\left\{\left(r(t)\left[x^{\prime}(t)\right]^{\gamma}\right)^{\prime \prime}+Q_{1}(t) x^{\gamma}(t)\right\} \operatorname{sgn} x(t) \leq 0
$$

has property (A) for some $k \in(0,1)$. Assuming the contrary, we admit that $\left(E_{2}\right)$ does not property $(\mathrm{A})$, that is, it possesses an eventually positive solution $x(t)$ satisfying $\left(N_{2}\right)$. We define have the following:

$$
w(t)=\frac{\left[r(t)\left[x^{\prime}(t)\right]^{\gamma}\right]^{\prime}}{x^{\gamma}(t)}>0
$$

Differentiating $w(t)$, one gets in view of $\left(E_{2}\right)$ that

$$
\begin{aligned}
w^{\prime}(t) & =\frac{\left[r(t)\left[x^{\prime}(t)\right]^{\gamma}\right]^{\prime \prime}}{x^{\gamma}(t)}-\gamma \frac{\left[r(t)\left[x^{\prime}(t)\right]^{\gamma}\right]^{\prime}}{x^{\gamma}(t)} \frac{x^{\prime}(t)}{x(t)} \\
& \leq Q_{1}(t)-\gamma w(t) \frac{x^{\prime}(t)}{x(t)} .
\end{aligned}
$$

On the other hand, using the monotonicity of $\left[r(t)\left[x^{\prime}(t)\right]^{\gamma}\right]^{\prime}$, we have

$$
\begin{aligned}
r(t)\left[x^{\prime}(t)\right]^{\gamma} \\
\quad \geq \int_{t_{0}}^{t}\left[r(s)\left[x^{\prime}(s)\right]^{\gamma}\right]^{\prime} \\
\geq\left[r(t)\left[x^{\prime}(t)\right]^{\gamma}\right]^{\prime}\left(t-t_{0}\right) \\
\geq \operatorname{et}\left[r(t)\left[x^{\prime}(t)\right]^{\gamma}\right]^{\prime},
\end{aligned}
$$

eventually; let us say that for $t \geq t_{1}$ or equivalently

$$
x^{\prime}(t) \geq\left(e t\left[r(t)\left[x^{\prime}(t)\right]^{\gamma}\right]^{\prime}\right)^{1 / \gamma} r^{-1 / \gamma}(t),
$$

where $\ell \in(0,1)$ will be specified latter. Setting the last inequality into (17), we obtain

$$
w^{\prime}(t) \leq-Q_{1}(t)-\gamma \ell^{1 / \gamma} w^{1+1 / \gamma}(t) \frac{t^{1 / \gamma}}{r^{1 / \gamma}(t)} .
$$

Integrating the last inequality from $t$ to $\infty$, we have

$$
w(t) \geq Q(t)+\gamma \ell^{1 / \gamma} \int_{t}^{\infty} w^{1+1 / \gamma}(s)\left(\frac{s}{r(s)}\right)^{1 / \gamma} \mathrm{d} s
$$

or

$$
\begin{aligned}
\frac{w(t)}{Q(t)} & \geq \\
1 & +\frac{\gamma \ell^{1 / \gamma}}{Q(t)} \int_{t}^{\infty}\left(\frac{s}{r(s)}\right)^{1 / \gamma} Q^{1+1 / \gamma}(s)\left(\frac{w(s)}{Q(s)}\right)^{1+1 / \gamma} \mathrm{d} s,
\end{aligned}
$$

eventually; let us say that $t \geq t_{1}$. Since $w(t)>Q(t)$, then

$$
\inf _{t \geq t_{1}} \frac{w(t)}{Q(t)}=\lambda \geq 1 \text {. }
$$

Thus,

$$
\frac{w(t)}{Q(t)} \geq 1+\frac{\gamma \ell^{1 / \gamma} \lambda^{1+1 / \gamma}}{Q(t)} \int_{t}^{\infty}\left(\frac{s}{r(s)}\right)^{1 / \gamma} Q^{1+1 / \gamma}(s) \mathrm{d} s .
$$

From (15), we see that there exist some $\ell \in(0,1)$ and some positive $\eta$, such that

$$
\frac{\ell^{1 / \gamma}}{Q(t)} \int_{t}^{\infty}\left(\frac{s}{r(s)}\right)^{1 / \gamma} Q^{1+1 / \gamma}(s) \mathrm{d} s>\eta>(\gamma+1)^{-(\gamma+1) / \gamma} .
$$


Combining (24) with (25), we have

$$
\frac{w(t)}{Q(t)} \geq 1+\gamma \lambda^{1+1 / \gamma} \eta
$$

Therefore,

$$
\lambda \geq 1+\gamma \lambda^{1+1 / \gamma} \eta>1+\gamma \lambda^{1+1 / \gamma}(\gamma+1)^{-(\gamma+1) / \gamma}
$$

or equivalently

$$
0>\frac{1}{\gamma+1}+\frac{\gamma}{\gamma+1}\left(\frac{\lambda}{\gamma+1}\right)^{1+1 / \gamma}-\frac{\lambda}{\gamma+1}
$$

This contradicts with the fact that the function

$$
g(\alpha)=\frac{1}{\gamma+1}+\frac{\gamma}{\gamma+1} \alpha^{1+1 / \gamma}-\alpha
$$

is positive for all $\alpha>0$, and we conclude that $x(t)$ cannot satisfy $\left(N_{2}\right)$; consequently, $\left(E_{1}\right)$ has property $(\mathrm{A})$.

In the following criteria, we utilize existing results known for differential equations without deviating arguments to provide new criteria for differential equations with mixed arguments.

Corollary 6. Assume that for some $k \in(0,1)$ the differential equation

$$
x^{\prime \prime \prime}(t)+\left\{\frac{q\left(\tau^{-1}(t)\right)}{\tau^{\prime}\left(\tau^{-1}(t)\right)}+k p(t) \frac{\sigma(t)}{t}\right\} x(t)=0
$$

enjoys property (A), then so does the equation with mixed arguments

$$
x^{\prime \prime \prime}(t)+q(t) x(\tau(t))+p(t) x(\sigma(t))=0 .
$$

Proof. By Theorem 4, $\left(E_{4}\right)$ enjoys property (A), provided that, for some $k \in(0,1)$, differential inequality

$$
\left[x^{\prime \prime \prime}(t)+\left\{\frac{q\left(\tau^{-1}(t)\right)}{\tau^{\prime}\left(\tau^{-1}(t)\right)}+k p(t) \frac{\sigma(t)}{t}\right\} x(t)\right] \operatorname{sgn} x(t) \leq 0
$$

has property (A). But by Corollary 1 in [16], this is equivalent to property $(\mathrm{A})$ of the corresponding differential equation $\left(E_{3}\right)$.

Corollary 7. Assume that at least one of the following conditions:

$$
\begin{aligned}
& \liminf _{t \rightarrow \infty} t^{2} \int_{t}^{\infty}\left(\frac{q\left(\tau^{-1}(s)\right)}{\tau^{\prime}\left(\tau^{-1}(s)\right)}+p(s) \frac{\sigma(s)}{s}\right) \mathrm{d} s>\frac{1}{3 \sqrt{3}}, \\
& \liminf _{t \rightarrow \infty} t \int_{t}^{\infty}\left(s \frac{q\left(\tau^{-1}(s)\right)}{\tau^{\prime}\left(\tau^{-1}(s)\right)}+p(s) \sigma(s)\right) \mathrm{d} s>\frac{2}{3 \sqrt{3}}
\end{aligned}
$$

Proof. It is known (see, e.g., $[12,15])$ that

$$
\liminf _{t \rightarrow \infty} t^{2} \int_{t}^{\infty} P(s) \mathrm{d} s>\frac{1}{3 \sqrt{3}}
$$

guarantees property $(\mathrm{A})$ of

$$
x^{\prime \prime \prime}(t)+P(t) x(t)=0 .
$$

The rest follows from Corollary 6 , where constant $k \in(0,1)$ is eliminated due to the sharp inequality of the used criterion.

We support our results by several illustrative examples.

\section{Examples}

Example 8. Consider the third-order nonlinear differential equation

$$
\left(t^{-1}\left(x^{\prime}(t)\right)^{3}\right)^{\prime \prime}+\frac{a}{t^{6}} x^{3}(\delta t)+\frac{b}{t^{6}} x^{3}(\lambda t)=0,
$$

where $a, b>0,0<\delta \leq 1$, and $\lambda \geq 1$. Simple computation shows that here (15) reduces to

$$
\delta^{5} a+k b \lambda^{4}>\left(\frac{5}{4}\right)^{4}
$$

for some $k \in(0,1)$, which is equivalent to

$$
\delta^{5} a+b \lambda^{4}>\left(\frac{5}{4}\right)^{4}
$$

and this by Theorem 4 guarantees that $\left(E_{x 1}\right)$ enjoys property (A).

Example 9. Consider the third-order differential equation with mixed arguments

$$
x^{\prime \prime \prime}(t)+\frac{a}{t^{3}} x(\delta t)+\frac{b}{t^{3}} x(\lambda t)=0,
$$

where $a, b>0,0<\delta \leq 1$, and $\lambda \geq 1$. Both conditions of Corollary 7 reduce to

$$
\delta^{2} a+b \lambda>\frac{2}{3 \sqrt{3}}
$$

which guarantees property $(\mathrm{A})$ of $\left(E_{x 2}\right)$.

Our results take into account the value of advanced argument, which can be seen in the following example.

Example 10. Consider the third-order nonlinear differential equation

$$
x^{\prime \prime \prime}(t)+\frac{a}{t^{3}} x(\delta t)+\frac{b}{t^{4}} x\left(t^{2}\right)=0,
$$

where $a, b>0,0<\delta<1$. Both conditions of Corollary 7 reduce to

$$
\delta^{2} a+b>\frac{2}{3 \sqrt{3}}
$$

which yields property $(\mathrm{A})$ of $\left(E_{x 3}\right)$. 


\section{Conclusion and Discussion}

In the paper, we presented new comparison theorems for studying the properties of third-order differential equations with mixed arguments through those of the corresponding differential equations without deviating arguments. The presented technique permits to extend immediately the results known for an equation without deviating arguments to a more general equation with advanced and delay arguments. The results obtained have been supported by several illustrative examples.

\section{Acknowledgment}

This work was supported by S.G.A. KEGA Grant no. 020TUKE-4/2012.

\section{References}

[1] R. P. Agarwal, S. R. Grace, and D. O'Regan, Oscillation Theory for Difference and Functional Differential Equations, Kluwer Academic, Dordrecht, The Netherlands, 2000.

[2] B. Baculíková and J. Džurina, "Oscillation of third-order neutral differential equations," Mathematical and Computer Modelling, vol. 52, no. 1-2, pp. 215-226, 2010.

[3] B. Baculíková and J. Džurina, "Oscillation of third-order neutral differential equations," Mathematical and Computer Modelling, vol. 52, no. 1-2, pp. 215-226, 2010.

[4] B. Baculíková, J. Graef, and J. Džurina, "On the oscillation of higher order delay differential equations," Nonlinear Oscillations, vol. 15, pp. 13-24, 2012.

[5] B. Baculíková and J. Džurina, "Oscillation of third-order nonlinear differential equations," Applied Mathematics Letters, vol. 24, no. 4, pp. 466-470, 2011.

[6] B. Baculíková and J. Džurina, "Oscillation of third-order functional differential equations," Electronic Journal of Qualitative Theory of Differential Equations, vol. 43, pp. 1-10, 2010.

[7] B. Baculíková and J. Džurina, "Comparison theorems for the third order delay trinomial differential equations," Advances in Difference Equations, vol. 2010, Article ID 160761, 12 pages, 2010.

[8] B. Baculíková, "Properties of third-order nonlinear functional differential equations with mixed arguments," Abstract and Applied Analysis, vol. 2011, Article ID 857860, 15 pages, 2011.

[9] B. Baculíková, J. Džurina, and Y. V. Rogovchenko, "Oscillation of third order trinomial delay differential equations," Applied Mathematics and Computation, vol. 218, no. 13, pp. 7023-7033, 2012.

[10] B. Baculíková and J. Džurina, "Property (A) and oscillation of third order differential equations with mixed arguments," Funkcialaj Ekvacioj, vol. 55, pp. 239-253, 2012.

[11] B. Baculíková, E. M. Elabbasy, S. H. Saker, and J. Džurina, "Oscillation criteria for third-order nonlinear differential equations," Mathematica Slovaca, vol. 58, no. 2, pp. 201-220, 2008.

[12] J. Džurina, "Comparison theorems for nonlinear ODEs," Mathematica Slovaca, vol. 42, no. 3, pp. 299-315, 1992.

[13] L. H. Erbe, Q. Kong, and B. G. Zhang, Oscillation Theory for Functional Differential Eqautions, Marcel Dekker, New York, NY, USA, 1995.

[14] J. K. Hale, Theory of Functional Differential Equations, Springer, New York, NY, USA, 1977.
[15] I. T. Kiguradze and T. A. Chanturia, Asymptotic Properties of Solutions of Nonatunomous Ordinary Differential Equations, Kluwer Academic, Dordrecht, The Netherlands, 1993.

[16] T. Kusano and M. Naito, "Comparison theorems for functionaldifferential equations with deviating arguments," Journal of the Mathematical Society of Japan, vol. 33, no. 3, pp. 509-532, 1981.

[17] G. S. Ladde, V. Lakshmikantham, and B. G. Zhang, Oscillation Theory of Differential Equations with Deviating Arguments, Marcel Dekker, New York, NY, USA, 1987.

[18] W. E. Mahfoud, "Oscillation and asymptotic behavior of solutions of $N$ th order nonlinear delay differential equations," Journal of Differential Equations, vol. 24, no. 1, pp. 75-98, 1977.

[19] A. Zafer, "Oscillation criteria for even order neutral differential equations," Applied Mathematics Letters, vol. 11, no. 3, pp. 21-25, 1998.

[20] T. Li, Z. Han, P. Zhao, and S. Sun, "Oscillation of even-order neutral delay differential equations," Advances in Difference Equations, vol. 2010, Article ID 184180, 9 pages, 2010.

[21] Ch. G. Philos, "A new criterion for the oscillatory and asymptotic behavior of delay differential equations," Académie Polonaise des Sciences, Série des Sciences Mathématiques, vol. 39, pp. 61-64, 1981. 


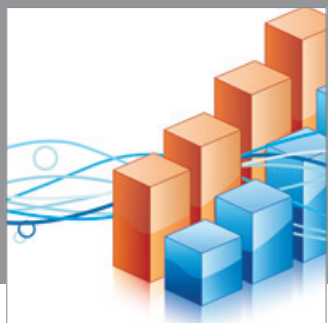

Advances in

Operations Research

mansans

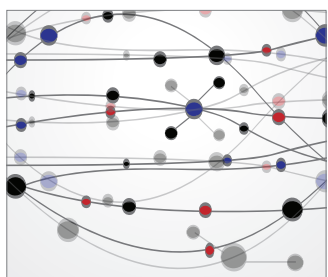

The Scientific World Journal
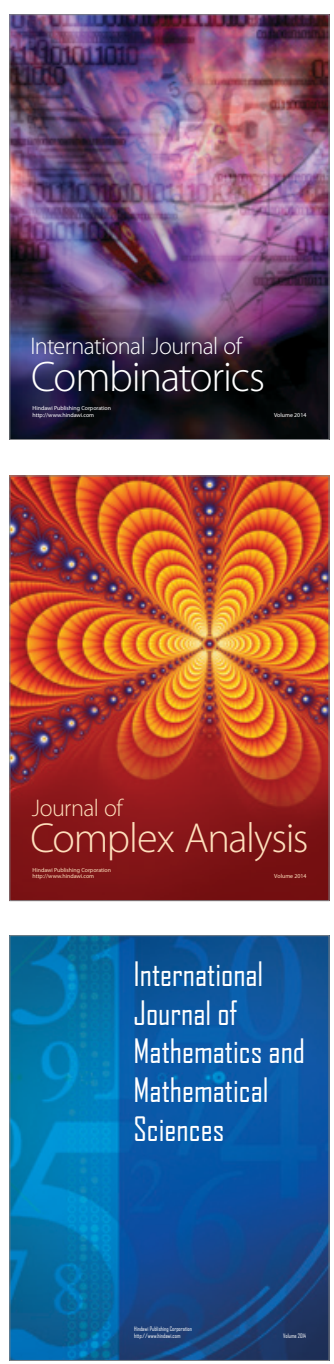
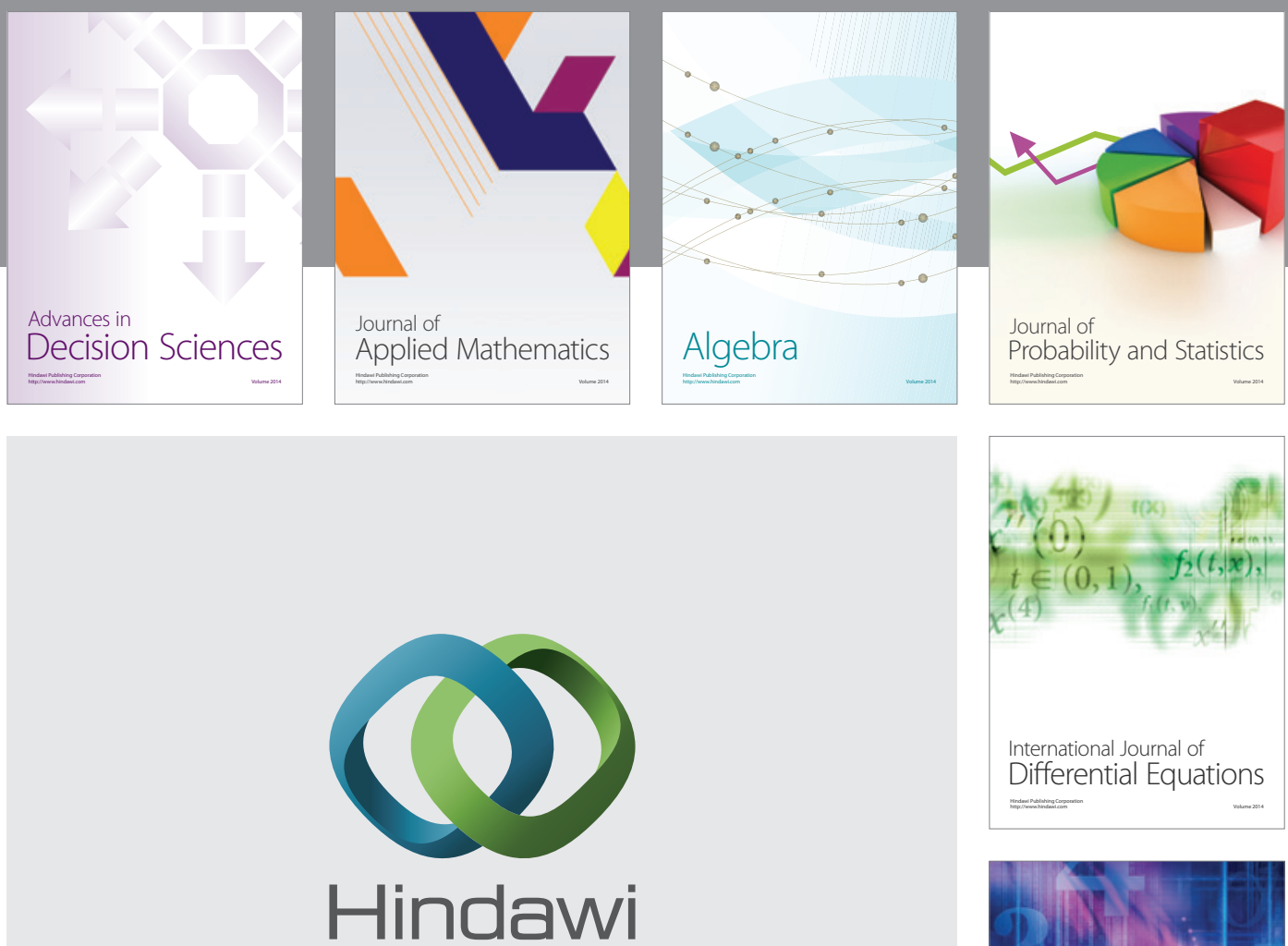

Submit your manuscripts at http://www.hindawi.com
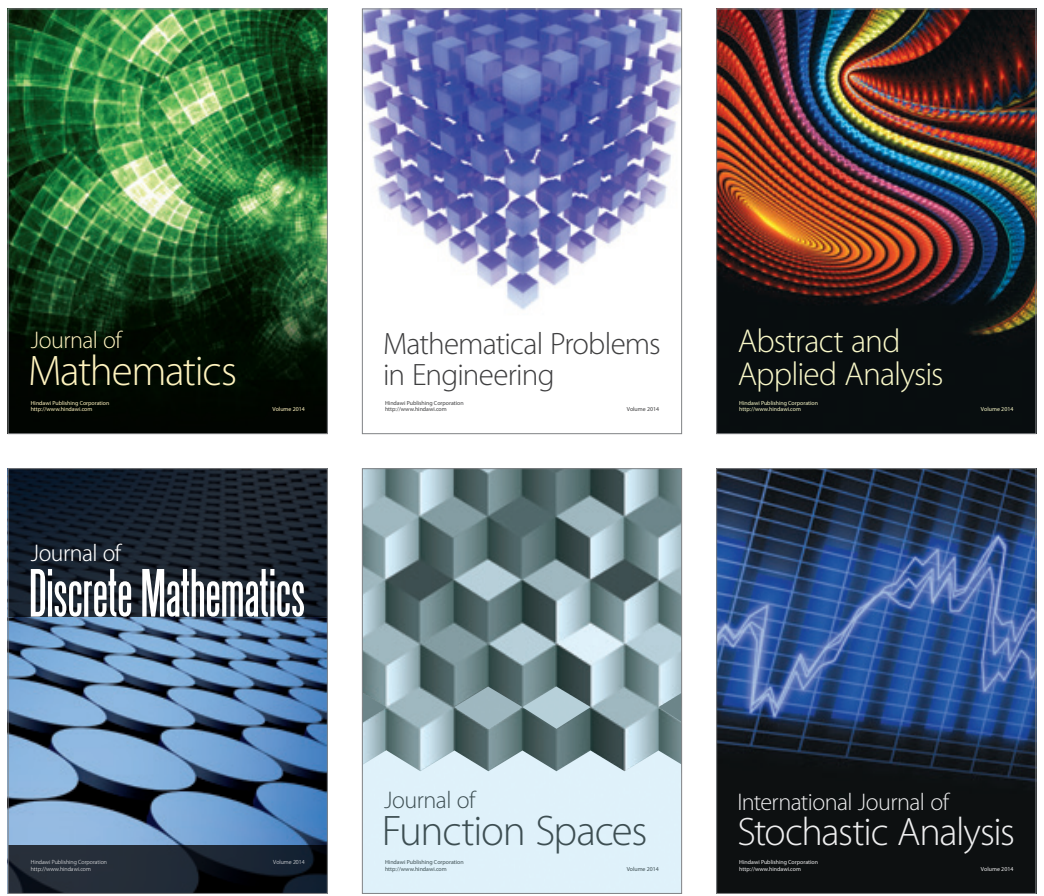

Journal of

Function Spaces

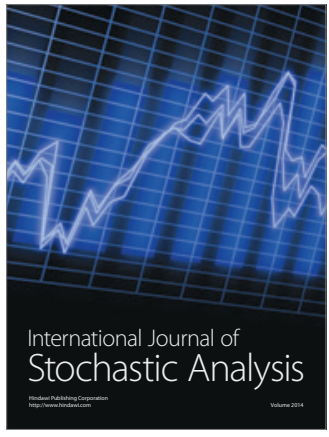

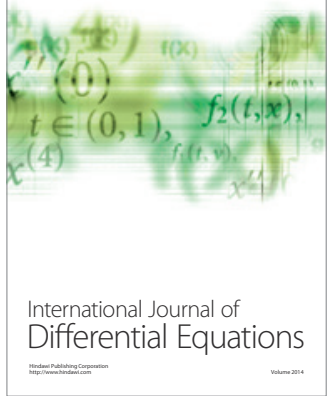
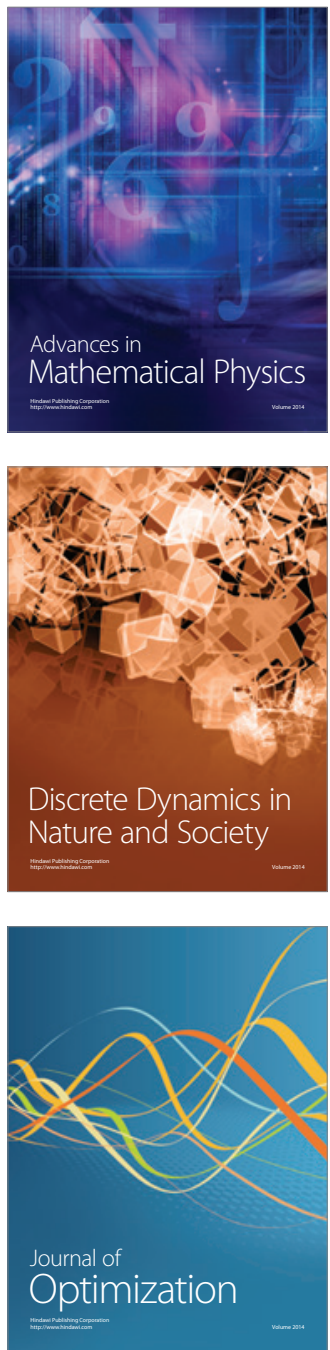